\title{
Immune amegakaryocytic thrombocytopenia of the newborn: association with anti-HLA-A2
}

\author{
D I K EVANS \\ From The Royal Manchester Children's Hospital, Pendlebury
}

SUMMARY Three newborn babies with congenital immune amegakaryocytic thrombocytopenia are described. Two were siblings. The mothers' sera showed antibody against both pooled random platelets and lymphocytes of type HLA-A2. Two babies had transient thrombocytopenia and leucopenia and made a full haematological recovery. One baby died aged four hours having had no haematological investigations during life. Histological examination of the bone marrow in all three babies (two by trephine biopsy; one at necropsy) confirmed a deficiency of megakaryocytes. It is suggested that anti-HLA-A2 may cause neonatal thrombocytopenia by depressing megakaryocytes instead of, or in addition to, any direct effect on platelets.

Immune thrombocytopenic purpura of the newborn is associated with maternal antibodies against specific platelet antigens (isoimmune) or antibodies common to all platelets (autoimmune). In the case of isoimmunity the mother lacks a common platelet antigen, usually $\mathrm{Pl}^{\mathrm{A} 1}$ (also known as $\mathrm{Zw}^{\mathrm{a}}$ ), which is present in $98 \%$ of the population. She becomes sensitised by her fetus, who carries the antigen, inherited from the father. In the case of autoimmunity the mother has, or has had, autoimmune thrombocytopenia, systemic lupus erythematosus, or some similar disease. In both types of neonatal purpura the bone marrow is hypercellular with increased megakaryocytes. It is unusual for immune neonatal thrombocytopenia to be associated with depression or absence of bone marrow megakaryocytes. When neonatal amegakaryocytic thrombocytopenia does occur, it is not immune mediated and is nearly always associated with the thrombocytopenia-absent radius syndrome (TAR), congenital infection, or leukaemia. "Authentic congenital hypoplastic thrombocytopenia without other abnormalities is rare, if indeed it occurs at all". ${ }^{1}$

The specificity of platelet antigens and antibodies was first elucidated by Shulman et al. ${ }^{2}$ Confirmation of any specificity still remains difficult as few laboratories offer a service; so many antibodies are not tested; and early reports omit any mention of specificity. Even now, there are few cases reported in which antibodies other than anti-PI ${ }^{\mathrm{A} 1}$ are mentioned. The HLA system is much better understood. Not all

Accepted for publication 24 September 1986
HLA antigens are carried on platelets, but the antigen HLA-A2 is carried by granulocytes, lymphocytes, and platelets and was formerly known as $\operatorname{PIGrLy}^{\mathbf{B} 1}{ }^{2}$ One of the cases reported by Shulman et $a l^{2}$ had thrombocytopenia with depressed bone marrow megakaryocytes due to anti-PlGrLy ${ }^{\text {B1 }}$ (anti-HLAA2). This antibody is an accepted but extremely rare cause of neonatal thrombocytopenia, ${ }^{1}$ but no other cases have been reported in which it has caused thrombocytopenia with megakaryocyte depression.

\section{Material and methods}

Bone marrow was aspirated from the posterior iliac crest with a Salah needle or 18 gauge lumbar puncture needle. Trephine biopsy was done with a paediatric Jamshidi needle. Platelet antibodies were studied with a modified enzyme immunoassay. ${ }^{3}$ Platelets were counted with a Baker 810 platelet analyser. HLA antibodies were detected with the microcytotoxicity test. $^{4}$

\section{Case reports}

\section{CASE 1}

The second baby of healthy parents who work on a farm was born at term, after a healthy pregnancy, by normal delivery. He weighed $2.76 \mathrm{~kg}$. The liquor was stained with meconium and blood and the placenta was unhealthy. He had a glandular hypospadias and a widespread petechial rash. The haemoglobin concentration was $6.4 \mathrm{~g} / \mathrm{dl}$, reticulocytes $2.1 \%$, platelets $19 \times 10^{9} / 1$, and blood glucose less than $0.5 \mu \mathrm{mol} / 1$. The anaemia was attributed to haemorrhage and the 
hypoglycaemia to asphyxia. A direct antiglobulin test was negative. An umbilical catheter was inserted and he was treated with $50 \%$ dextrose and fresh frozen plasma. Later an exchange transfusion was given. A lumbar puncture excluded meningitis, but the field was blood-stained and cerebral haemorrhage was diagnosed. He remained irritable but the haemoglobin concentration rose to $12.7 \mathrm{~g} / \mathrm{dl}$ and the platelets to $32 \times 10^{9} / 1$. He was monitored through an indwelling umbilical arterial catheter. Over the next 48 hours the haemoglobin concentration fell to $8.4 \mathrm{~g} / \mathrm{dl}$ and platelets to $18 \times 10^{9} / 1$. A computed tomography scan showed ventricular dilatation attributed to a bleed in the left temporoparietal area. A further blood transfusion raised the haemoglobin concentration to $12.0 \mathrm{~g} / \mathrm{dl}$. A platelet transfusion was also given and the platelet count rose to $110 \times 10^{9} / 1$. The next day he developed paraplegia and a paralysed bladder, attributed to spinal cord embolism. He was more alert. He was transferred to this hospital, where the liver was noted transiently to be two fingerbreadths enlarged and the spleen was tipped. Haemoglobin concentration was $10 \cdot 1 \mathrm{~g} / \mathrm{dl}$ and platelets $120 \times 10^{9} / 1$. White cells were $3.6 \times 10^{9} / 1$ (polymorphs $66 \%$, lymphocytes $31 \%$, monocytes $1 \%$, eosinophils $2 \%$ ). Bone marrow was aspirated with difficulty.

There was erythroid hyperplasia and a reduction of granulocytes and lymphocytes. Only very scanty megakaryocytes were seen. Two further aspirations showed a slight increase of histiocytes but essentially similar findings. Trephine biopsy from the posterior iliac crest showed no megakaryocytes. The baby's blood was weakly positive for platelet antibodies by enzyme immunoassay. The platelet count progressively fell again to a minimum of $5 \times 10^{9} / 1$ on day 12 . A second platelet transfusion was given that increased the platelet count to $183 \times 10^{9} / 1$, which fell to $65 \times 10^{9} / 1$ on day 19 . Thereafter the platelet count rose spontaneously to normal values.

Initial studies also included tests for toxoplasmosis which were positive on both mother and baby's blood. The baby's dye test rose from 1/4096 immediately after birth to $1 / 32000$ six months later; IgM ELISA antibodies were positive shortly after delivery at 53 enzyme immunoassay units and fell to 3 enzyme immunoassay units. The findings indicated congenital infection with Toxoplasma gondii, but no clinical evidence of the disease was detected. A computed tomography scan on day 4 had shown subarachnoid and intraventricular haemorrhage, and a repeat scan at five months showed left cerebral atrophy and ventricular dilatation. There was no calcification. Retinal examination was unremarkable, including an examination under anaesthetic at seven months.

The mother's blood, when tested against pooled platelets, was positive for platelet antibodies by enzyme immunoassay, and weak platelet antibodies were also detected in the baby's blood. The mother's blood was also positive for anti-HLA2 to a titre of $1 / 16$, and a possible anti-B27 to $1 / 4$. The mother was HLA A3, A29, B7, B12, and the baby was HLA-A2, B7, B27. Antibody tests for cytomegalovirus showed only weak decaying maternal antibody, and no viruses were isolated from three samples of urine. The white cell count was less than $4.5 \times 10^{9} / 1$ on days 4 to 6 , and the haemoglobin fell to $8.4 \mathrm{~g} / \mathrm{dl}$ on day 9 . No further transfusion was given at this hospital, and the baby returned to the referring hospital with a haemoglobin concentration of $10.3 \mathrm{~g} / \mathrm{dl}$, white cell count $7 \cdot 1 \times 10^{9} / 1$ (polymorphs $57 \%$, lymphocytes $40 \%$, monocytes $3 \%$ ) and platelets $183 \times 10^{9} / 1$ on day 12 . There was no recurrence of purpura, and apart from two isolated platelet counts of $40 \times 10^{9} / 1$ and $66 \times 10^{9} / 1$ on days 21 and 36 , the blood count remained normal. The paraplegia persists at the time of writing.

\section{CASE 2}

This female baby was born after her mother's second pregnancy, at full term. Both parents were healthy. The first pregnancy had produced normal twins. The baby died after four hours with severe purpura and pallor. The necropsy showed only anaemia and intrapulmonary haemorrhage; but the necropsy sections showed only very occasional megakaryocytes in the bone marrow, mostly with pycnotic non-segmented nuclei. No platelet count was recorded.

\section{CASE 3}

This baby was her parents' fourth child, the offspring of her mother's third pregnancy, and the sister of case 2. She was born at full term after a normal delivery and weighed $3.37 \mathrm{~kg}$. She had generalised purpura at birth. Haemoglobin concentration was $18.8 \mathrm{~g} / \mathrm{dl}$, white cell count $8.1 \times 10^{9} / 1$, and platelets $11 \times 10^{9} / 1$. The platelets were noted to be large on the film. Chromosome analysis showed a normal female $46 \mathrm{XX}$. She was transfused with two units of random platelets and given $3 \mathrm{mg} / \mathrm{kg} /$ day of prednisone. Twenty four hours later she was transferred to this hospital with a platelet count of $108 \times 10^{9} / 1$ that fell to 73 and then to $27 \times$ $10^{9} / 1$ on the second and third days of life. The first white cell count was normal, but a low count of $3 \cdot 1 \times$ $10^{9} / 1$ (neutrophils $79 \%$, lymphocytes $12 \%$ ) was found on day 3. Total white cell counts on days 4,5 , and 6 $\left(5 \cdot 3,4 \cdot 5\right.$, and $\left.4.6 \times 10^{9} / 1\right)$ remained in the low range for a newborn without infection. There was no further extension of the purpura and no untoward effects. The platelet count rose gradually to $147 \times 10^{9} / 1$ on day 12 (white cells $7.7 \times 10^{9} / 1$; polymorphs $67 \%$, lymphocytes $15 \%$, monocytes $17 \%$, eosinophils $1 \%$ ). By then the purpura had resolved completely and the 
prednisone was stopped. Thereafter she made normal progress.

The mother's blood was tested for antiplatelet activity by enzyme immunoassay against pooled platelets, the husband's platelets, and autologous platelets. All tests initially yielded negative results. No antibodies were found to random platelets by $\mathrm{C}^{\mathbf{1 4}}$ serotonin release. Lymphocytotoxic antibodies were detected with anti-HLA2 specificity to a titre of over $1 / 16$. Tests for antibodies to thyroglobulin and thyroid microsomes, antinuclear factor, smooth muscle, mitochondria, reticulin and gastric parietal cells were negative. She was group $O$ rhesus (D) positive. Her lymphocytes showed a balanced reciprocal B-C translocation: $46 \mathrm{XX}, \mathrm{t}(5 ; 10)(\mathrm{p} 151 ; \mathrm{q} 22)$. This was considered to be innocuous and had previously been detected in the normal twins. The affected baby had normal chromosomes. Seven months after delivery the serum was retested for antiplatelet antibodies by enzyme immunoassay: a positive result was obtained. The mother's HLA type was A3, A29, B12, B14 and the baby was A2, A3, B14, Bw16. The baby's blood group was $\mathrm{O}$ rhesus (D) negative. The mother's serum was negative for anti-PIA .

Both mothers were seen again three years later. The HLA antibodies were still present, but no platelet antibody was present. Both children were haematologically normal.

\section{Discussion}

It is rare for neonatal immune thrombocytopenia to be associated with reduced or absent bone marrow megakaryocytes in the absence of other congenital abnormalities or infection. An increase of megakaryocytes is the usual finding. ${ }^{5}$ Even though neonatal death occurs in $12 \%$ of cases of isoimmune neonatal purpura, ${ }^{5}$ too few necropsies have been reported to show the incidence of megakaryocyte hypoplasia.

The role of anti-HLA-A2 in these cases seems to have been critical. In 1962 Shulman et al ${ }^{6}$ described maternal isoimmunisation caused by a platelet antigen which they called $\mathrm{PP}^{\mathrm{B}}$. They later found that it was shared with granulocytes and lymphocytes and so renamed it PlGrLy ${ }^{B 1}$. It is now known to be the transplantation antigen HLA-A2 and is present in $48 \%$ of the population. They also suggested that the antigen might be present on megakaryocytes and that the antibody could cause amegakaryocytic thrombocytopenia, as one of their cases with anti-PIGrLy ${ }^{\text {B1 }}$ was thus affected. Another similar case was due to anti-PIGrLy $^{\mathrm{C} 1}$ (now recognised as anti-HLA-A7). ${ }^{5}$ These are the only antibodies of the HLA system that have been definitely implicated in maternal isoimmunisation against platelets. ${ }^{1}$
Von dem Borne et $a l^{7}$ tested 38 mothers of thrombocytopenic newborns. In 33 platelet specific antibodies were found. Three sera also contained HLA antibodies. Twenty nine of the $33(88 \%)$ were anti$\mathrm{Zw}^{\mathrm{a}}$ (identical with anti-PI ${ }^{\mathrm{A}}{ }^{1}$ ). The three HLA antibodies reacted against $B 8, A 3$, and $B 7+B 17$. On the other hand, maternal HLA antibodies rarely cause illness in the baby. On screening 1507 women Sharon and $\mathrm{Amar}^{8}$ found HLA antibodies in $495(33 \%)$. None had babies with thrombocytopenia.

Shulman $e t a l^{2}$ infused anti-HLA-A2 into volunteers and produced a short lived leucopenia. This is not unexpected in view of the antibody's wider specificity, and may be the cause for the leucopenia seen in cases 1 and 3. The presence of leucopenia in addition to thrombocytopenia may indicate the presence of amegakaryocytic thrombocytopenia in the newborn.

Boggs $^{9}$ reported being unable to locate more than two dozen or so reports of congenital amegakaryocytic thrombocytopenia. Of these, many had other abnormalities and those without, where antibodies were associated, included only the two cases of Shulman et $a^{2}$ already mentioned, and one of Hugh-Jones et al, ${ }^{10}$ with platelet agglutinins in the baby's serum. Confirmation by trephine biopsy is virtually unknown. At a later age Boggs ${ }^{9}$ could detect only three cases, and one of these went on to develop polycythaemia. He postulated that an antibody affecting both platelets and megakaryocytes may be found eventually, perhaps the anti-HLA-A2 antibody described here.

The role of Toxoplasma antibodies in case 1 calls for comment. There is a clear association between amegakaryocytic neonatal thrombocytopenia and congenital rubella, syphilis, and cytomegalovirus infection. It is not unreasonable to suppose that intrauterine infection with another agent such as toxoplasmosis might also cause depression of megakaryocytes. The antibody changes in case 1 clearly indicated a congenital infection. There were no signs whatever of clinical disease, however, even after a computed tomography scan of the brain and retinoscopy under anaesthetic. Furthermore, the thrombocytopenia rapidly resolved without specific treatment for toxoplasmosis. A low platelet count is not a recognised complication of congenital toxoplasmosis. It seems probable that the toxoplasmosis is not related to the thrombocytopenia.

Case 2, who died with neonatal purpura, had depressed bone marrow megakaryocytes, and although no platelet count was recorded, it is probable that thrombocytopenia was the cause of death. It is interesting that this mother's antibody led to megakaryocyte depression in two babies. It suggests either a genetic susceptibility of the marrow to respond in 
this way, or more likely, a specific property of this anti-HLA-A2 antibody. Interestingly, anti-HLA-A2 was present at birth, but anti-platelet activity was not detected until seven months later.

Neonatal thrombocytopenia progressing to later aplastic anaemia has also been described. ${ }^{11}{ }^{12}$ It is one of the constitutional bone marrrow aplasias. Symptoms often develop in the first week of life, and generalised bone marrow failure develops after an interval ranging from several months to $12 \frac{1}{2}$ years. The disease is distinct from Fanconi's anaemia, as there are no associated physical defects or chromosomal abnormalities. It is also distinct from the syndrome of thrombocytopenia with absent radii (TAR syndrome). It is now three years since the last of these babies was first seen and there has been no further blood disorder. It seems unlikely that they will develop this complication.

This report confirms that hypoplastic thrombocytopenia certainly occurs without other abnormalities, in contrast to Miller's statement, ${ }^{1}$ but it is extremely rare. Fortunately, it is self limiting and capable of recovering fully in two or three weeks. With present methods of support, the prognosis should be good.

I am grateful to Mr J Cunliffe for the platelet antibody studies, Miss G Morell for the HLA antibody tests, and to Dr D Donnai for details of family history for cases 2 and 3.

\section{References}

1 Miller DR, Baehner RL, McMillan CW, Miller LP. Blood diseases in infancy and childhood. Fifth ed. St Louis: CV Mosby, 1984;820-5.

2 Shulman NR, Marder VJ, Hiller MC, Collier EM. Platelet and leucocyte isoantigens and their antibodies. Serologic, physiologic and clinical studies. Progr Hematol 1964;4:222-304.

3 Gudino M, Miller WV. Application of the enzyme-linked immunospecific assay (ELISA) for the detection of platelet antibodies. Blood 1981;57:32-7.

4 Dick HM, Crighton BW. Tissue typing techniques. London: Churchill Livingstone, 1972.

5 Pearson HA, Shulman NR, Marder VJ, Cone TR Jr. Isoimmune neonatal thrombocytopenic purpura: clinical and therapeutic considerations. Blood 1964;23:154-77.

6 Shulman NR, Aster RH, Pearson HA, Hiller MC. Immunoreactions involving platelets. VI. Reactions of maternal isoantibodies responsible for neontal purpura. Differentiation of a second platelet antigen system. J Clin Invest 1962;41: 1059-69.

7 Von dem Borne AEG Kr, Vanleeuwen EF, Von Riesz LE, Van Boxtel CJ, Engelfriet CP. Neonatal allo-immune thrombocytopenia; detection and characterisation of the responsible antibodies by the platelet immunofluorescence test. Blood 1981;57:649-56.

8 Sharon R, Amar A. Maternal anti-HLA antibodies and neontal thrombocytopenia. Lancet 1981;i:1313.

9 Boggs DR. Amegakaryocytic thrombocytopenia. Am J Hematol 1985;20:413-6.

10 Hugh-Jones K, Manfield PA, Brewer HF. Congenital thrombocytopenic purpura. Arch Dis Child 1960;35:146-52.

11 O'Gorman Hughes DW. The varied pattern of aplastic anaemia in childhood. Aust Paediatr J 1966;2:228-36.

12 O'Gorman Hughes DW. Aplastic anaemia in childhood. III. Constitutional aplastic anaemia and related cytopenias. Med J Aust 1974;1:519-26.

Requests for reprints to: Dr DIK Evans, The Royal Manchester Children's Hospital, Pendlebury, Manchester M27 1HA, England. 